\title{
Epigenetic Regulation of Filaggrin Gene Expression in Human Epidermal Keratinocytes
}

\author{
Jaehyouk Lee $^{1,2}$, Ara Jang ${ }^{1,2}$, Seong Jun Seo ${ }^{3, *}$, Soon Chul Myung ${ }^{1,2, *}$ \\ ${ }^{1}$ Bio-Integration Research Center for Nutra-Pharmaceutical Epigenetics, Chung-Ang University, \\ Departments of ${ }^{2}$ Urology and ${ }^{3}$ Dermatology, Chung-Ang University College of Medicine, Seoul, Korea
}

Background: Loss-of-function mutations in the filaggrin gene (FLG), which encodes an epidermal protein crucial for the formation of a functional skin barrier, have been identified as a major predisposing factor in the etiopathogenesis of atopic dermatitis (AD). Recent reports of relatively low frequencies of FLG-null mutations among specific ethnic groups with AD necessitated analysis of the epigenetic regulation which may control FLG expression without altering its DNA sequence. Objective: The study aimed to identify DNA methylation- dependent regulation of FLG expression. Methods: Quantitative polymerase chain reaction was performed to determine the restoration of FLG mRNA expression in normal human epidermal keratinocyte (NHEK) cells after treatment with epigenetic modulating agents. Bisulfite genomic sequencing and pyrosequencing analyses of the FLG promoter region were conducted to identify the citical $\mathrm{CpG}$ sites relevant to FLG expression. We performed small-scale pilot study for epidermal tissues obtained from Korean patients with severe AD. Results: We here show that DNA methylation in the FLG with non-CpG island promoter is responsible for the transcriptional regulation of FLG in undifferentiated NHEK cells. The methylation frequencies in a single $\mathrm{CpG}$ site of the FLG

Received August 16, 2019, Revised November 26, 2019, Accepted for publication December 2, 2019

*These authors have equally contributed to the article.

Corresponding author: Soon Chul Myung, Department of Urology, Chung-Ang University College of Medicine, 84 Heukseok-ro, Dongjak-gu, Seoul 06974, Korea. Tel: 82-2-6299-1808, Fax: 82-2-6294-1406, E-mail: uromyung@ cau.ac.kr

ORCID: https://orcid.org/0000-0002-6727-5450

This is an Open Access article distributed under the terms of the Creative Commons Attribution Non-Commercial License (http://creativecommons. org/licenses/by-nc/4.0) which permits unrestricted non-commercial use, distribution, and reproduction in any medium, provided the original work is properly cited.

Copyright $(c)$ The Korean Dermatological Association and The Korean Society for Investigative Dermatology promoter were significantly higher in lesional epidermis than those in matched nonlesional epidermis of subjects with severe AD. Conclusion: Our in vitro and clinical studies point to this unique CpG site as a potential DNA methylation marker of FLG, which can be a promising therapeutic target in the complications of filaggrin-related skin barrier dysfunction as well as in AD. (Ann Dermatol 32(2) 122 129, 2020)

\section{-Keywords-}

Atopic dermatitis, DNA methylation, Keratinocyte

\section{INTRODUCTION}

Profilaggrin, filaggrin (filament-aggregating protein), and their proteolyzed amino acids are multifunctional protein components contributing to formation of the intact stratum corneum barrier ${ }^{1}$. According to the "outside-inside" view, atopic dermatitis (AD) is a common chronic skin disease that is characterized by abnormal permeability barrier owing to the deficiency of filaggrin ${ }^{2}$. Since two prevalent loss-of-function (LOF) mutations (p.R501X and c.2282del4) of the filaggrin gene ( $F L G$ ) were identified in the European populations with ichthyosis vulgaris (IV) and atopic ecze$\mathrm{ma}^{3,4}$, a comprehensive analytical strategy for screening of FLG variants has been implemented in the cohorts with not only IV or AD but also secondary allergic diseases ${ }^{5}$. Although various FLG-null mutations have been revealed in the selected Asian populations (including Japanese ${ }^{6,7}$, Chinese $^{8,9}$, Singaporean Chinese ${ }^{10}$, Taiwanese ${ }^{11}$, and Korean $^{12,13}$ ), the prevalence and mutation rates of FLC-null alleles are different from European cohorts with IV or AD. Additionally, FLG LOF variants were found to be less common in patients of African ancestry with AD compared with European and Asian patients ${ }^{14,15}$. A recent study re- 
vealed FLG mutations in $22.2 \%$ of African Americans with IV/AD ${ }^{16}$, whereas no FLG LOF mutations were detected in South African amaXhosa patients with $\mathrm{AD}^{17}$. Besides the genetic background predisposing to skin barrier dysfunction, non-genetic alterations of FLG seem to be correlated with AD pathogenesis in non-European populations, which have shown relatively low frequencies of FLG-null mutations.

An epigenetic trait is defined as a stably heritable phenotype resulting from changes in a chromosome without alterations in the DNA sequence ${ }^{18}$. Epigenetic mechanisms including DNA methylation, histone modifications, and non-coding RNAs have been implicated in a huge variety of human diseases correlating with gene-environment interactions ${ }^{19,20}$. Methylation of the cytosine base within a $\mathrm{CpG}$ dinucleotide context in gene promoters is generally associated with transcriptional repression ${ }^{21,22}$. Aberrant DNA methylation and subsequent silencing of a tumor suppressor gene have also been demonstrated in skin tumors, such as basal cell carcinoma, and in autoimmunityrelated skin disorders ${ }^{23}$. Recently, epigenetic alterations have been proposed as an alternative mechanism of predisposition to $A D$ in people with wild-type FLG. Ziyab et al. $^{24}$, for example, reported that DNA methylation at the single CpG site in the FLG gene body showed a significant interaction with FLG LOF variants on the risk for eczema. Nonetheless, epigenetic factors that may account for the deficiency of filaggrin and its degradation products in AD remain elusive. We hypothesized that epigenetic changes at the FLG gene promoter might contribute to modulation of FLG expression in human epidermal keratinocytes and might be associated with one of the etiopathogenic mechanisms that trigger the deficit of filaggrin in the affected epidermis of AD patients.

\section{MATERIALS AND METHODS}

\section{Ethics statement}

This study was conducted according to the Declaration of Helsinki Principles and was approved by the Institutional Review Board of the Chung-Ang University Hospital (IRB no. C2015258 [1716]). All patients gave their written informed consent to participate in the study.

\section{Cell culture and drug treatment}

Normal human epidermal keratinocyte (NHEK) cells were obtained from the PromoCell (Heidelberg, Germany). NHEK cells were grown in Keratinocyte Growth Medium 2 (PromoCell) supplemented with Keratinocyte Growth Medium 2 SupplementMix (PromoCell). All cells were maintained at $37^{\circ} \mathrm{C}$ in a humidified incubator at $5 \% \mathrm{CO}_{2}$.
We treated the undifferentiated NHEK cells with 2'-deoxy5-azacytidine (DAC) for 72 hours and with trichostatin A (TSA) for 24 hours. DAC and TSA were obtained from Sigma-Aldrich (St. Louis, MO, USA).

\section{Tissue samples}

Punch biopsy specimens were collected from the paired non-lesional and lesional skin of patients with severe AD $(n=10)$. Epidermal samples were isolated under a dissecting microscope. Genomic DNA extraction was performed using the QIAamp DNA Mini Kit (Qiagen, Hilden, Germany).

\section{Quantitative polymerase chain reaction}

As described in detail previously ${ }^{25}$, total RNA was isolated from the cell lines using the RNeasy Mini Kit (Qiagen) following the manufacturer's instructions. cDNA synthesis for real-time two-step reverse transcription polymerase chain reaction (PCR) was performed on $1 \mu \mathrm{g}$ of total RNA using the QuantiTect Reverse Transcription Kit (Qiagen), and then $1 \mu \mathrm{l}$ of diluted cDNA was utilized for cycling reactions using the Rotor-Gene SYBR Green PCR Kit (Qiagen). The amplification and quantitative analysis were performed on the Rotor-Gene Q 5plex HRM system (Qiagen). Thermal cycling was conducted using the default conditions of the Rotor-Gene $\mathrm{Q}$ Series Software (Qiagen), which consisted of 5 minutes at $95^{\circ} \mathrm{C}$ followed by 40 rounds of 5 seconds at $95^{\circ} \mathrm{C}$ and 10 seconds at $60^{\circ} \mathrm{C}$. The transcript level measured was normalized to the results of a QuantiTect Primer Assay (Qiagen) for ACTB (encoding $\beta$-actin), which served as an internal control.

\section{In vitro methylated reporter assay}

As described in detail previously ${ }^{25}$, a 928-bp fragment harboring four CpG sites located at the $5^{\prime}$-end region of FLG (NC_ 000001.11), a 5' Nsil site, and a 3' HindIII site was synthesized and cloned into the pCpGfree-basic-Lucia reporter plasmid (InvivoGen, San Diego, CA, USA) that codes for a secreted coelenterazine-utilizing variant of luciferase. Briefly, $5 \mu \mathrm{g}$ of the Lucia reporter plasmid was methylated using $12 \mathrm{U}$ of M.Sssl CpG methyltransferase (New England BioLabs, Ipswich, MA, USA) in vitro at $37^{\circ} \mathrm{C}$ for 20 hours. After purification with the DNA Clean \& Concentrato $^{\text {TM }}-5$ Kit (Zymo Research, Irvine, CA, USA), methylation of the plasmid was verified by bisulfite pyrosequencing of the selected $\mathrm{CpG}$ units in the FLG promoter (Supplementary Fig. 1). Next, HEK293T cells were transiently transfected with unmethylated or methylated reporter plasmids by means of the jetPEI reagent (Polyplustransfection, New York, NY, USA). Luciferase activity of the Lucia reporter in the supernatant of the cells at 72 
hours after the transfection was measured using the QUANTILuc assay reagent (InvivoGen). In each experiment, the cells were cotransfected with the pCMV-CLuc 2 Control Plasmid (New England BioLabs), which encodes a secreted variant of Cypridina luciferase, as a normalization control. Luminescence induced by Cypridina luciferase in the cell supernatant was determined using the BioLux Cypridina Luciferase Assay Kit (New England BioLabs). Reporter activity was normalized by calculating the ratio of Lucia to CLuc activities.

\section{DNA methylation analyses}

For bisulfite genomic sequencing analysis, as described in detail previously ${ }^{25}$, genomic DNA was extracted from the cell lines using the QIAamp DNA Mini Kit. Bisulfite modification of the genomic DNA was performed using the EZ DNA Methylation-Lightning ${ }^{\mathrm{TM}}$ Kit (Zymo Research). The bisulfite-converted genomic DNA was amplified using primer sets specific to the proximal promoter region of FLC (positions -738 to -162 upstream of the transcription start site (TSS), which is denoted as +1 in Supplementary Fig. 2) containing four $\mathrm{CpG}$ units. The sequences of the PCR primers used were as follows: forward (5'-AGAAGGAAGAG TATGTGGAATATG-3') and reverse (5'- CAACAACCTATA TTTACTTCCCAAC-3'). Cycling conditions were as follows: initial denaturation at $94^{\circ} \mathrm{C}$ for 10 minutes; $45 \sim 50$ cycles of denaturation at $94^{\circ} \mathrm{C}$ for 30 seconds, annealing at $56^{\circ} \mathrm{C}$ for 30 seconds, and extension at $72^{\circ} \mathrm{C}$ for $30 \mathrm{sec}$ onds; and the final extension at $72^{\circ} \mathrm{C}$ for 10 minutes. PCR products were purified and subcloned into the pGEM-T Easy Vector (Promega, Madison, WI, USA) for subsequent sequencing analysis. The nucleotide sequences of $12 \sim 15$ independent clones were analyzed.

Furthermore, the bisulfite-modified genomic DNA obtained from normal individuals and AD patients was amplified using primer sets specific to two key CpG sites within the FLG promoter. The sequences of the PCR primers used were as follows: CpG 4 site, forward (5'-GAA GGAAGAGTATGTGGAATATGT-3'), 5'-biotinylated reverse (5'-CACTAAAAACATAAATTTAATTAAACAAAACTC-3'), and sequencing (5'- TGTGGAATATGTTTTTGATGTTA-3'); CpG 2 site, forward (5'-AGATGGAATATATAGATTAAAAAA GAATA-3'), 5'-biotinylated reverse (5'-TAAATACACTTCC TAATCCTTATCTCT-3'), and sequencing (5'-TTTTTATTAT AAATTGAATTTTAAG-3'). Pyrosequencing reactions were performed using the PyroMark Gold Q96 Reagents (Qia gen) and quantitative analysis was conducted on the PyroMark Q96 ID platform (Qiagen).

\section{Statistical analysis}

Data were presented as mean \pm standard error of the mean and analyzed by Student's t-test. To determine significant differences in the quantitative methylation data generated by pyrosequencing measurements, comparisons between nonlesional and lesional tissue samples were made using the Wilcoxon signed-rank test. Differences with a $p<0.05$ were considered statistically significant. Statistical analyses were conducted using the IBM SPSS Statistics ver. 20 software (IBM Corp., Armonk, NY, USA).

\section{RESULTS \\ DNA methylation-dependent regulation of FLG expression in NHEK cells}

To test whether epigenetic regulation of FLG takes place in NHEK cells, we first examined the restoration of FLG expression in undifferentiated NHEK cells after treatment with either the DNA methyltransferase inhibitor DAC or the histone deacetylase inhibitor TSA (Fig. 1A). The DAC-induced DNA demethylation and TSA-mediated histone acetylation triggered a significant increase in FLC mRNA levels in undifferentiated NHEK cells. These data implied that the loss of FLG expression in undifferentiated NHEK cells correlated with epigenetic silencing involving both DNA methylation and histone modifications.

Next, to determine whether DNA methylation in the 5 '-end region of FLG was important for modulating the transcriptional activity of the core promoter, we conducted an in vitro methylated reporter assay (Fig. 1B). We synthesized an FLG fragment containing the non-CpG island (CGI) promoter and four $\mathrm{CpG}$ dinucleotide units therein (Fig. 1C). In vitro methylation was performed after cloning this fragment into a CpG-free luciferase plasmid to assess the role of CpG methylation in FLG transcription. HEK293T cells transfected with the methylated FLG reporter plasmid showed markedly decreased luciferase activity as compared with the cells transfected with the unmethylated FLC plasmid. The magnitude of the reduction between unmethylated control and methylated plasmid was approximately $90 \%$, suggesting that DNA methylation directly led to transcriptional silencing of FLG with non-CGI promoter.

To identify the critical $\mathrm{CpG}$ sites important for regulating FLC expression in undifferentiated NHEK cells, we performed bisulfite genomic sequencing of the proximal promoter region of FLG. We determined the methylation profiles of the four $\mathrm{CpG}$ dinucleotides (i.e., $\mathrm{CpG} 1$ to 4 ) located in the FLG promoter, assigning the first CpG dinucleotide to a site 185 bp upstream of FLG TSS (CpG 1). As shown in Fig. $1 \mathrm{C}$, the methylation profile of the FLG promoter revealed a substantial difference in methylation frequency at both the CpG 2 and CpG 4 loci $(-410$ and -702 
A

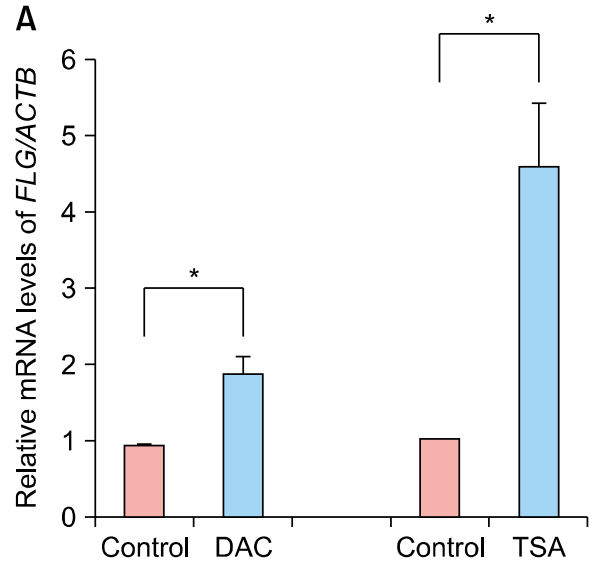

B

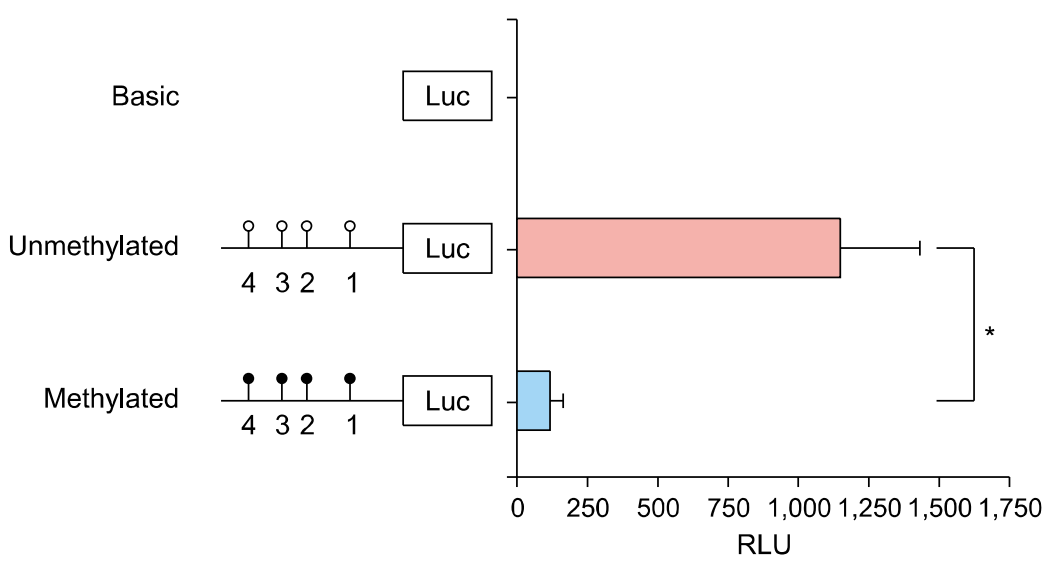

C

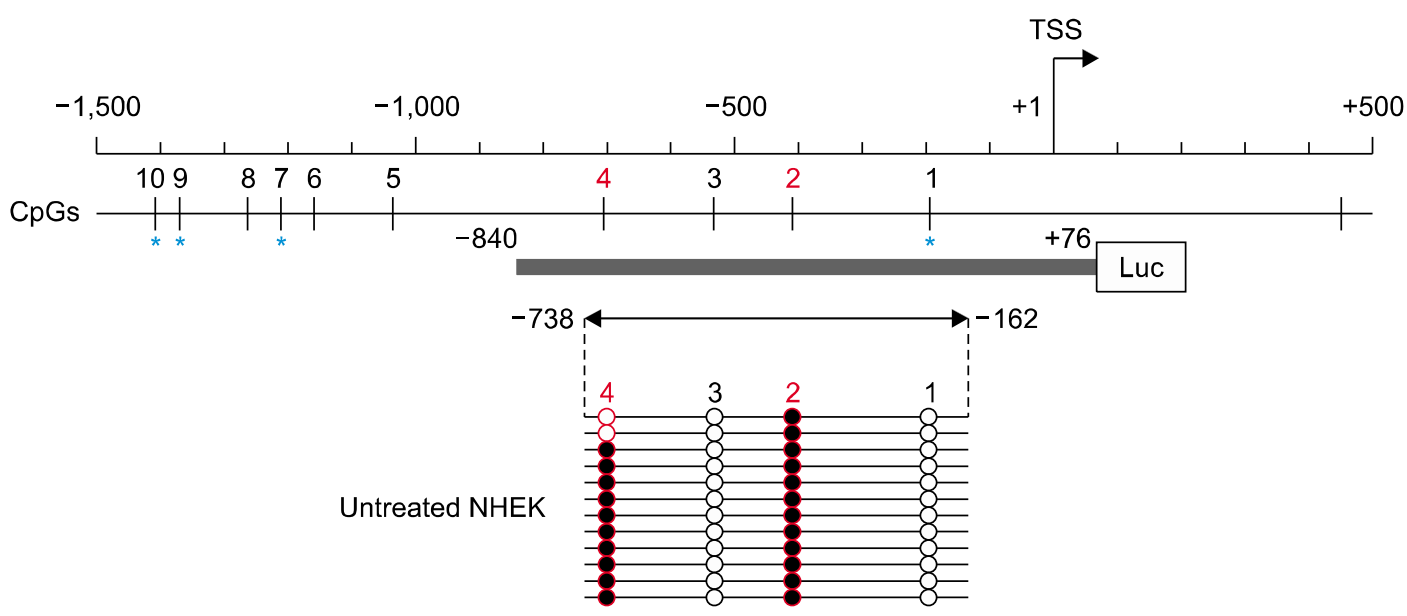

FLG

(NC_000001.11)

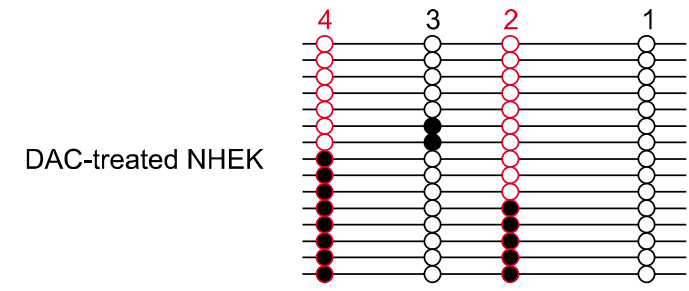

Fig. 1. Epigenetic regulation of filaggrin gene (FLG) expression in undifferentiated normal human epidermal keratinocyte (NHEK) cells. (A) Quantitative polymerase chain reaction was performed to determine the restoration of $F L G$ mRNA expression in undifferentiated NHEK cells after treatment with DNA demethylating agent 2'-deoxy-5-azacytidine (DAC) or histone deacetylase inhibitor trichostatin A (TSA). (B) A promoter reporter assay of the $5^{\prime}$-end region of FLG in HEK293T cells using the pCpGfree-basicLucia reporter plasmid. The synthesized fragment contained four $\mathrm{CpG}$ dinucleotide sites (represented by lollipops). (C) Bisulfite sequencing analysis of the FLG promoter harboring CpG 1 through CpG 4 sites in undifferentiated NHEK cells. Circles represent stand-alone CpG sites, and each row represents the DNA sequence of an individual clone. Unmethylated and methylated CpG units are depicted as white and black circles, respectively. The blue asterisk indicates the location of CpG ID in the Illumina HumanMethylation450 BeadChip: CpG 1, cg19855573; CpG 7, cg13447818; CpG 9, cg10500702; CpG 10, cg26390526. ACTB: beta-Actin, RLU: relative light unit, TSS: transcription start site. ${ }^{*} p<0.05$.

bp upstream of the FLG TSS, respectively) in the DAC-treated NHEK cells, when compared to control cells. These findings indicate that the site-specific CpG dinucleotides within the FLG promoter might be preferentially affected by a DNA demethylating agent in undifferentiated NHEK cells.

\section{CpG methylation frequencies in the FLG promoter were higher in lesional tissues than in non-lesional tissues of $A D$ subjects}

To evaluate whether the epigenotyping for FLG by using of the putative DNA methylation marker (i.e., CpG 2 and 
CpG 4 dinucleotide units within the FLG promoter) was applicable to the epidermal tissues from AD patients, we performed a small-scale pilot study comparing methylation differences of these $\mathrm{CpG}$ loci between non-lesional and lesional epidermis obtained from Korean patients with severe AD (Eczema Area Severity Index > 15; $n=10$; Table 1). After the triplicate experiments of bisulfite pyrosequencing for each pairs of matched samples, we observed that CpG 2 sites were characterized by overall higher methylation frequencies in lesional tissues than in matched nonlesional tissues (Fig. 2A). Although the differential methylation status at the CpG 4 unit was detected during the bisulfite genomic sequencing of FLC in undifferentiated NHEK cells, no significant correlation was observed in the quantitative methylation analysis between nonlesional and lesional epidermal tissue samples of AD subjects (Fig. 2B). Nonetheless, a statistically significant association of methylation frequencies was shown by a single $\mathrm{CpG}$ unit, i.e., CpG 2, within the FLG promoter $(p=0.0343)$ in the skin biopsy samples categorized into non-lesional and lesional samples from the AD patients (Fig. 2B).

\section{DISCUSSION}

Epigenetic alterations in AD, including histone modifications and miRNAs, have been discovered in a variety of genes except $F L G^{26,27}$. The $5^{\prime}$-end region of $F L G$ is devoid of any defined CGI (length $\geq 200 \mathrm{bp}$, Guanine-Cytosine content $\geq 50 \%$, and observed-to-expected CpG ratio $\left[\right.$ Obs $\left._{\mathrm{CpG}} / \operatorname{Exp}_{\mathrm{CpG}}\right] \geq 0.6$ ) (Supplementary Fig. 2$)^{28}$. The potential involvement of variable methylation in the non-CGI promoter of FLG has been mostly overlooked. In contrast to genes with CGIs at their TSSs, substantial fluctuations occur in the promoter methylation levels of genes that are

Table 1. Clinical characteristics of patients with atopic dermatitis

\begin{tabular}{|c|c|c|c|c|}
\hline Subject & $\begin{array}{c}\text { Sex/age } \\
(y r)\end{array}$ & EASI & $\operatorname{lgE}$ & $\begin{array}{c}\text { Biopsy site } \\
\text { (NL/L) }\end{array}$ \\
\hline 1 & $M / 22$ & 19.0 & 2,386 & Back/popliteal \\
\hline 2 & $M / 47$ & 17.8 & NA & Forearm/popliteal \\
\hline 3 & $\mathrm{M} / 42$ & 25.2 & NA & Thigh/abdomen \\
\hline 4 & $\mathrm{~F} / 19$ & 20.5 & 206 & Thigh/neck \\
\hline 5 & $M / 17$ & 24.6 & $>5,000$ & Abdomen/antecubital \\
\hline 6 & $\mathrm{M} / 56$ & 18.4 & 805 & Back/neck \\
\hline 7 & $M / 32$ & 16.0 & $>5,000$ & Thigh/neck \\
\hline 8 & $M / 45$ & 24.0 & $>5,000$ & Back/neck \\
\hline 9 & $M / 31$ & 17.9 & 1,784 & Back/back \\
\hline 10 & $M / 39$ & 19.3 & NA & Back/flank \\
\hline
\end{tabular}

EASI: Eczema Area Severity Index, IgE: immunolobulin E, NL: non-lesion, L, lesion, M: male, F: female, NA: not available.
CpG-poor at the $\mathrm{TSS}^{22}$. Because $\mathrm{CpG}$-poor regulatory regions tend to acquire a low methylation state when occupied by transcription factors ${ }^{29,30}$, DNA methylation at non-CGI promoters have high information content about the ongoing activity of transcription factors ${ }^{21}$. Several studies have shown that no significant promoter methylation is associated with FLG expression in AD cases $^{24,31}$ and in buccal cells ${ }^{32}$. Herein, we focused on the CpG sites that were not examined in previous epigenetic analyses, because the methylation array-based studies on FLG in AD subjects could not have included all the differentially methylated CpG loci in the non-CGI promoter of FLG. Noticeably, non-CGI promoters, which are generally hypermethylated, remain transcriptionally active regardless of their methylation state ${ }^{33,34}$, but our findings demonstrate that DNA methylation of only four CpG sites directly suppress the non-CGl promoter of FLG. Moreover, significant differences were observed in the methylation frequency of the single CpG site between non-lesional and lesional epidermis obtained from Korean patients with severe AD, suggesting that the possible epigenetic alterations in vivo might depend on the methylation status at this site-specific CpG unit within the FLG promoter.

It has been previously found that the levels of filaggrin breakdown products, such as trans-urocanic acid and pyrrolidone-5-carboxylic acid, are significantly reduced in patients with $\mathrm{AD}$ without FLG-null mutations ${ }^{17,35}$. Our results support the insight that epigenetic alterations may account for discordant phenotypes of patients with a skin disease who are wild-type on the prevalent mutations of the disease $^{36}$. Because a given cytosine can either be completely methylated or unmethylated, 'variable methylation' is the outcome of averaging these binary states ${ }^{22}$. Hence, what is measured for a given epidermal sample at a given $\mathrm{CpG}$ site, i.e., the CpG 2 unit within the FLG promoter, is the percentage of epidermal cells that are methylated. Considering the significant differences in the methylation frequencies among the dermatologist-diagnosed AD samples, this key CpG 2 unit in the FLG promoter can serve as an AD-associated DNA methylation biomarker. Indeed, a single CpG site showing differential methylation between different disease states is known as a methylation variable position (MVP), which can be considered as the epigenetic equivalent of a single nucleotide polymorphism ${ }^{37,38}$. This might suggest that the methylation frequency at any given MVP may serve as a novel epigenetic signature for physiological and pathologic status ${ }^{39,40}$. Thus, our observations also suggest that the CpG 2 unit within the FLG promoter might serve as an MVP candidate of filaggrindeficient skin phenotype.

In conclusion, we provided evidence for one of the mech- 

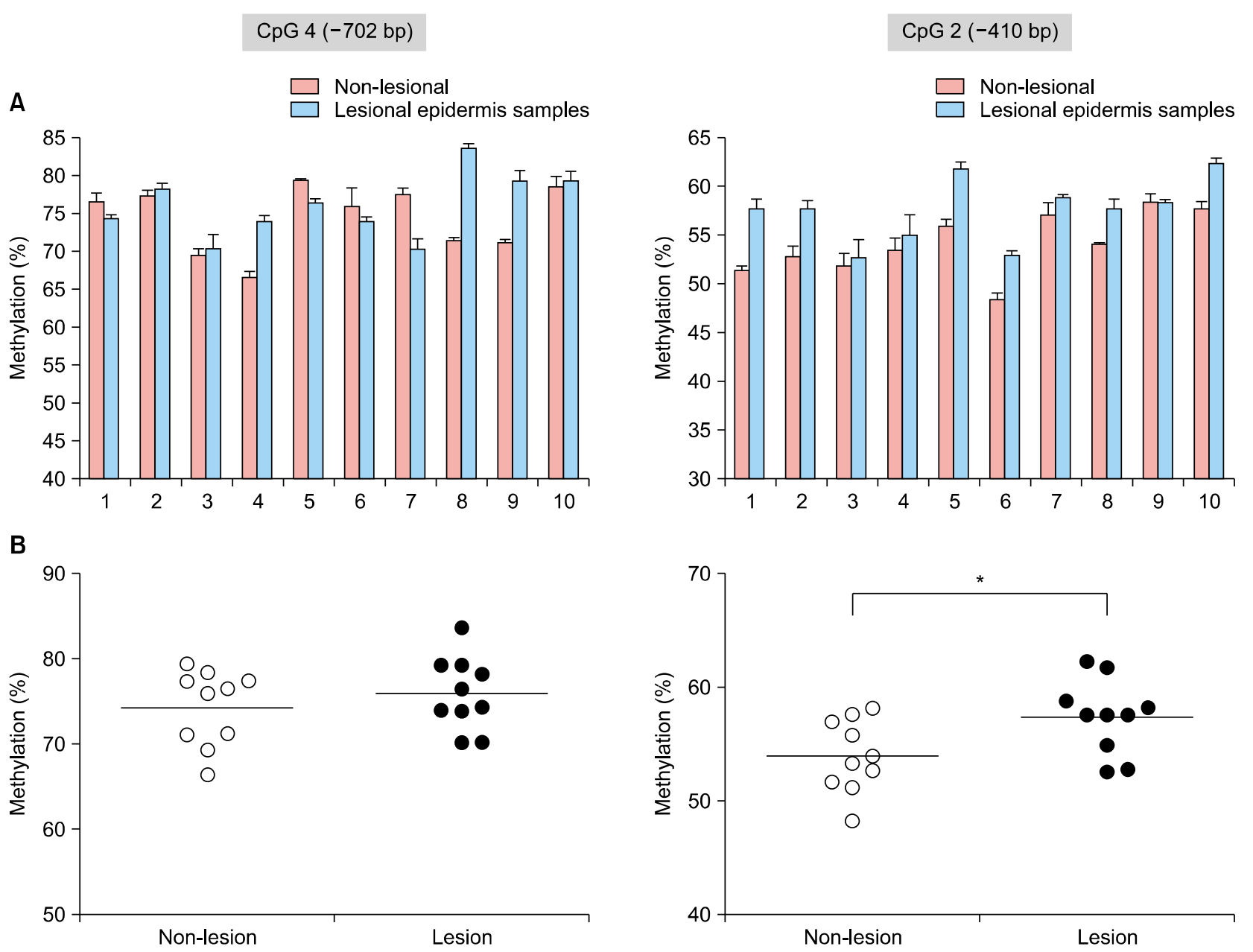

Fig. 2. Quantitative methylation analysis of the $\mathrm{CpG} 4$ and $\mathrm{CpG} 2$ dinucleotide units in patients with atopic dermatitis (AD) ( $n=10$ ). (A) Bisulfite pyrosequencing analysis of the CpG $4(-702 \mathrm{bp})$ and $\mathrm{CpG} 2(-410 \mathrm{bp})$ sites in the FLG promoter region. (B) Significant association between non-lesional and lesional tissues was found in the methylation frequencies of the CpG 2 unit. The methylation frequencies of the CpG 4 and $\mathrm{CpG} 2$ units were determined using triplicate experiments of bisulphite pyrosequencing. ${ }^{*} p<0.05$.

anisms via which significant contributors to filaggrin deficiency work in the lesional skin of patients with AD. To extrapolate the plausible correlation between epigenotype of FLG and genetic background of affected individuals and controls, future studies should involve a larger AD cohort where the genotyping for the FLG-null mutations is performed beforehand. Furthermore, our finding may have more promising clinical relevance in conjunction with FLG-genotyping studies, which include diseases of various severity.

\section{ACKNOWLEDGMENT}

The authors thank Chung-Ang University Hospital Biomedical Research Institute for kindly offering research equipment.

This work was supported by a grant (714001-07) from the
Center for Industrialization of Natural Nutraceuticals through the Agriculture, Food and Rural Affairs Research Center Support Program, Ministry of Agriculture, Food and Rural Affairs, Republic of Korea.

\section{SUPPLEMENTARY MATERIALS}

Supplementary data can be found via http://anndermatol.org/ src/sm/ad-32-122-s001.pdf.

\section{CONFLICTS OF INTEREST}

The authors have nothing to disclose.

\section{ORCID}

Jaehyouk Lee, https://orcid.org/0000-0001-8161-9953 
Ara Jang, https://orcid.org/0000-0002-8388-1688

Seong Jun Seo, https://orcid.org/0000-0003-2915-839X

Soon Chul Myung, https://orcid.org/0000-0002-6727-5450

\section{REFERENCES}

1. Brown SJ, McLean WH. One remarkable molecule: filaggrin. J Invest Dermatol 2012;132(3 Pt 2):751-762.

2. Elias PM, Steinhoff M. "Outside-to-inside" (and now back to "outside") pathogenic mechanisms in atopic dermatitis. J Invest Dermatol 2008;128:1067-1070.

3. Smith FJ, Irvine AD, Terron-Kwiatkowski A, Sandilands A, Campbell LE, Zhao $Y$, et al. Loss-of-function mutations in the gene encoding filaggrin cause ichthyosis vulgaris. Nat Genet 2006;38:337-342.

4. Palmer CN, Irvine AD, Terron-Kwiatkowski A, Zhao Y, Liao $\mathrm{H}$, Lee SP, et al. Common loss-of-function variants of the epidermal barrier protein filaggrin are a major predisposing factor for atopic dermatitis. Nat Genet 2006;38:441-446.

5. Sandilands A, Terron-Kwiatkowski A, Hull PR, O'Regan GM, Clayton TH, Watson RM, et al. Comprehensive analysis of the gene encoding filaggrin uncovers prevalent and rare mutations in ichthyosis vulgaris and atopic eczema. Nat Genet 2007;39:650-654.

6. Nomura T, Sandilands A, Akiyama M, Liao H, Evans AT, Sakai $K$, et al. Unique mutations in the filaggrin gene in Japanese patients with ichthyosis vulgaris and atopic dermatitis. J Allergy Clin Immunol 2007;119:434-440.

7. Hamada T, Sandilands A, Fukuda S, Sakaguchi S, Ohyama $B$, Yasumoto $S$, et al. De novo occurrence of the filaggrin mutation p.R501X with prevalent mutation c.3321delA in a Japanese family with ichthyosis vulgaris complicated by atopic dermatitis. J Invest Dermatol 2008;128:1323-1325.

8. Zhang X, Liu S, Chen X, Zhou B, Liu D, Lei G, et al. Novel and recurrent mutations in the filaggrin gene in Chinese patients with ichthyosis vulgaris. Br J Dermatol 2010;163: 63-69.

9. Zhang H, Guo Y, Wang W, Shi M, Chen X, Yao Z. Mutations in the filaggrin gene in Han Chinese patients with atopic dermatitis. Allergy 2011;66:420-427.

10. Chen H, Common JE, Haines RL, Balakrishnan A, Brown SJ, Goh CS, et al. Wide spectrum of filaggrin-null mutations in atopic dermatitis highlights differences between Singaporean Chinese and European populations. Br J Dermatol 2011; 165:106-114.

11. Hsu CK, Akiyama M, Nemoto-Hasebe I, Nomura T, Sandilands A, Chao SC, et al. Analysis of Taiwanese ichthyosis vulgaris families further demonstrates differences in FLG mutations between European and Asian populations. Br J Dermatol 2009;161:448-451.

12. Kim EJ, Jeong MS, Li K, Park MK, Lee MK, Yoon Y, et al. Genetic polymorphism of FLG in Korean ichthyosis vulgaris patients. Ann Dermatol 2011;23:170-176.

13. Park J, Jekarl DW, Kim Y, Kim J, Kim M, Park YM. Novel FLG null mutations in Korean patients with atopic dermatitis and comparison of the mutational spectra in Asian populations. J Dermatol 2015;42:867-873.

14. Winge MC, Bilcha KD, Liedén A, Shibeshi D, Sandilands A, Wahlgren $C F$, et al. Novel filaggrin mutation but no other loss-of-function variants found in Ethiopian patients with atopic dermatitis. Br J Dermatol 2011;165:1074-1080.

15. Margolis DJ, Gupta J, Apter AJ, Hoffstad O, Papadopoulos $M$, Rebbeck TR, et al. Exome sequencing of filaggrin and related genes in African-American children with atopic dermatitis. J Invest Dermatol 2014;134:2272-2274.

16. Polcari I, Becker L, Stein SL, Smith MS, Paller AS. Filaggrin gene mutations in African Americans with both ichthyosis vulgaris and atopic dermatitis. Pediatr Dermatol 2014;31: 489-492.

17. Thawer-Esmail F, Jakasa I, Todd G, Wen Y, Brown SJ, Kroboth K, et al. South African amaXhosa patients with atopic dermatitis have decreased levels of filaggrin breakdown products but no loss-of-function mutations in filaggrin. J Allergy Clin Immunol 2014;133:280-282.e1-e2.

18. Berger SL, Kouzarides T, Shiekhattar R, Shilatifard A. An operational definition of epigenetics. Genes Dev 2009;23: 781-783.

19. Jaenisch R, Bird A. Epigenetic regulation of gene expression: how the genome integrates intrinsic and environmental signals. Nat Genet 2003;33 Suppl:245-254.

20. Botchkarev VA, Gdula MR, Mardaryev AN, Sharov AA, Fessing MY. Epigenetic regulation of gene expression in keratinocytes. J Invest Dermatol 2012;132:2505-2521.

21. Schübeler D. Function and information content of DNA methylation. Nature 2015;517:321-326.

22. Jones PA. Functions of DNA methylation: islands, start sites, gene bodies and beyond. Nat Rev Genet 2012;13:484-492.

23. Li Y, Sawalha AH, Lu Q. Aberrant DNA methylation in skin diseases. J Dermatol Sci 2009;54:143-149.

24. Ziyab AH, Karmaus W, Holloway JW, Zhang H, Ewart S, Arshad SH. DNA methylation of the filaggrin gene adds to the risk of eczema associated with loss-of-function variants. J Eur Acad Dermatol Venereol 2013;27:e420-e423.

25. Lee J, Han JH, Jang A, Kim JW, Hong SA, Myung SC. DNA methylation-mediated downregulation of DEFB1 in prostate cancer cells. PLoS One 2016;11:e0166664.

26. Alaskhar Alhamwe B, Khalaila R, Wolf J, von Bülow V, Harb $\mathrm{H}$, Alhamdan $\mathrm{F}$, et al. Histone modifications and their role in epigenetics of atopy and allergic diseases. Allergy Asthma Clin Immunol 2018;14:39.

27. Bhardwaj N. MicroRNAs in atopic dermatitis: a review. J Transl Genet Genom 2017;1:15-22.

28. Gardiner-Garden M, Frommer M. CpG islands in vertebrate genomes. J Mol Biol 1987;196:261-282.

29. Stadler MB, Murr R, Burger L, Ivanek R, Lienert F, Schöler A, et al. DNA-binding factors shape the mouse methylome at distal regulatory regions. Nature 2011;480:490-495.

30. Ziller MJ, Gu H, Müller F, Donaghey J, Tsai LT, Kohlbacher $\mathrm{O}$, et al. Charting a dynamic DNA methylation landscape of the human genome. Nature 2013;500:477-481.

31. Rodríguez $E$, Baurecht $H$, Wahn AF, Kretschmer A, Hotze $M$, Zeilinger $S$, et al. An integrated epigenetic and transcriptomic analysis reveals distinct tissue-specific patterns of 
DNA methylation associated with atopic dermatitis. J Invest Dermatol 2014;134:1873-1883.

32. Tan HT, Ellis JA, Koplin JJ, Martino D, Dang TD, Suaini N, et al.; HealthNuts Study Investigators. Methylation of the filaggrin gene promoter does not affect gene expression and allergy. Pediatr Allergy Immunol 2014;25:608-610.

33. Weber $M$, Hellmann I, Stadler $M B$, Ramos L, Pääbo $S$, Rebhan $\mathrm{M}$, et al. Distribution, silencing potential and evolutionary impact of promoter DNA methylation in the human genome. Nat Genet 2007;39:457-466.

34. Meissner A, Mikkelsen TS, Gu H, Wernig M, Hanna J, Sivachenko A, et al. Genome-scale DNA methylation maps of pluripotent and differentiated cells. Nature 2008;454: 766-770

35. Kezic S, O'Regan GM, Yau N, Sandilands A, Chen $H$, Campbell LE, et al. Levels of filaggrin degradation products are influenced by both filaggrin genotype and atopic dermatitis severity. Allergy 2011;66:934-940.
36. Bataille $V$, Lens $M$, Spector TD. The use of the twin model to investigate the genetics and epigenetics of skin diseases with genomic, transcriptomic and methylation data. J Eur Acad Dermatol Venereol 2012;26:1067-1073.

37. Tost J, Dunker J, Gut IG. Analysis and quantification of multiple methylation variable positions in $\mathrm{CpG}$ islands by Pyrosequencing. Biotechniques 2003;35:152-156.

38. Rakyan VK, Down TA, Balding DJ, Beck S. Epigenome-wide association studies for common human diseases. Nat Rev Genet 2011;12:529-541.

39. Rakyan VK, Hildmann T, Novik KL, Lewin J, Tost J, Cox AV, et al. DNA methylation profiling of the human major histocompatibility complex: a pilot study for the human epigenome project. PLoS Biol 2004;2:e405.

40. Huang Q, Huang JF, Zhang B, Baum L, Fu WL. Methylation variable position profiles of $\mathrm{hMLH} 1$ promoter $\mathrm{CpG}$ islands in human sporadic colorectal carcinoma. Diagn Mol Pathol 2012;21:24-33. 